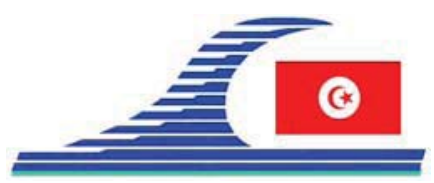

\author{
Conférence Méditerranéenne Côtière et Maritime \\ EDITION 1, HAMMAMET, TUNISIE (2009) \\ Coastal and Maritime Mediterranean Conference \\ Disponible en ligne - http://www.paralia.fr-Available online
}

\title{
Modélisation de la propagation de la houle dans la zone côtière de Tabarka-Berkoukech. Application du modèle STWAVE
}

\author{
Nabila HALOUANI ${ }^{1}$, Safa FATHALLAH ${ }^{1}$ Moncef GUEDDARI $^{1}$
}

1. Université de Tunis El Manar, Faculté des Sciences, Département de Géologie.

Campus Universitaire. 2092 El Manar, Tunis, Tunisie.nabila.fst@gmail.com

\section{Résumé :}

Le littoral tunisien est soumis à une pression croissante, suite au développement socioéconomique et à la gestion peu rationnelle de ce milieu, ce qui a entrainé, en de nombreux endroits, une érosion plus ou moins importante des côtes sableuses. Ce phénomène résulte de la combinaison de divers facteurs, induits à la fois par la nature et par l'homme, avec des modalités différentes dans le temps et dans l'espace.

Le littoral de Tabarka-Berkoukech, situé au Nord-Ouest de la Tunisie, n'échappe pas à ce phénomène de dégradation et connaît, par endroits, un recul important de sa ligne de rivage. La connaissance des conditions hydrodynamiques de cette frange littorale, en particulier celles qui sont liées à l'action de la houle, est nécessaire pour apprécier l'état de stabilité de son trait de côte et pour prévoir les moyens de sa protection.

Ce travail a pour objectif d'apprécier les phénomènes qui contrôlent la modification des caractéristiques de la houle (hauteur significative, période et direction), lors de sa propagation vers la côte, qui se traduit par une concentration ou par une dissipation d'énergie, ce qui est en faveur d'une érosion ou d'une sédimentation.

Pour atteindre cet objectif, une simulation de la propagation de la houle dans la zone côtière de Tabarka-Berkoukech a été faite à l'aide du modèle STWAVE (STeady-state spectral WAVE model), sous 1'interface SMS.9.2 (Surface-water-Modeling-System). Les principaux résultats obtenus nous ont permis de déterminer l'effet de la houle et des courants associés sur l'érosion côtière, en identifiant les zones à forte énergie (cap gréseux Borj Arif), où les iso-amplitudes de la houle se resserrent, et celles à faible énergie (plage el corniche), où les iso-amplitudes s'élargissent.

Les variations de la hauteur de la houle sont essentiellement causées par le phénomène de réfraction dans l'ensemble de secteur d'étude et, localement, par le phénomène de diffraction lié à la présence de l'île de Tabarka.

\section{Mots clés :}

Simulation de la houle - Modèle STWave - Erosion - Sable - Tabarka-Berkoukech

\section{Introduction}

La houle joue un rôle important dans le transport sédimentaire à l'approche de la côte, en particulier dans la zone de déferlement. En effet, lors de la propagation de la houle DOI: $10.5150 / \mathrm{cmcm} .2009 .026-2$ 
du large vers la côte, ses caractéristiques se trouvent fortement modifiées sous l'action combinée de multiples facteurs et phénomènes (influence de la bathymétrie, réfraction, déferlement, shoaling et diffraction...). Ces modifications se traduisent par une dissipation ou par une concentration d'énergie. Il est donc nécessaire pour la prédiction de la dynamique sédimentaire et pour l'analyse de la stabilité du trait de côte d'une plage sableuse, d'identifier et d'analyser les facteurs qui sont à l'origine de l'érosion ou de la sédimentation.

Le littoral de Tabarka-Berkoukech, situé au Nord-Ouest de la Tunisie, connaît, par endroits, un recul important de sa ligne de rivage. La connaissance des conditions hydrodynamiques de cette frange littorale, en particulier celles qui sont liées à l'action de la houle, est nécessaire pour apprécier l'état de stabilité de son trait de côte et pour prévoir les moyens de sa protection.

\section{Matériels et Méthodes}

\subsection{Présentation du secteur d'étude}

La frange côtière de Tabarka-Berkoukech, est limitée à l'Ouest par l'île de Tabarka et à l'Est par l'embouchure de l'oued Berkoukech (figure 1). La morphologie de ce littoral est dominée par une plage sableuse, large de plusieurs décamètres, interompue par des pointements rocheux (OUESLATI, 1994). La partie sous marine est marquée par la présence des barres d'avant côte, festonnées et non rythmiques. La topographie sous marine est caractérisée par une pente de 1,6\% entre 0 et $-5 \mathrm{~m}$ (zone de déferlement). Trois cours d'eau débouchent dans le littoral Tabarka-Berkoukech : l'oued El Kébir, l'oued Bouterfess et l'oued Berkoukech. Ils contribuent, en partie, aux apports solides et en bilan sédimentaire. La faible amplitude de la marée dans la zone d'étude, avec des valeurs comprises entre 45 et $60 \mathrm{~cm}$ (HP, 1995), qualifie le littoral Tabarka-Berkoukech de côte microtidale où les houles des secteurs Nord-Ouest et Nord-Est jouent un rôle majeur dans la morphodynamique des plages.

\subsection{Méthodologie}

Pour atteindre les objectifs visés, une simulation de la propagation de la houle dans la zone côtière de Tabarka-Berkoukech a été faite à l'aide du modèle STWAVE (STeadystate spectral WAVE model), (SMITH et al., 2001), sous l'interface SMS.9.2 (Surfacewater-Modeling-System). Ce modèle résout l'équation de conservation spectroangulaire d'énergie de la houle par la méthode de différences finies et prend en compte les phénomènes de réfraction, de shoaling et de diffraction, ainsi que la dissipation d'énergie dû au déferlement et au frottement de fond.

Les simulations numériques de la houle sur le littoral Tabarka-Berkoukech ont été réalisées en considérant deux conditions des houles dominantes, avec les valeurs suivantes de la hauteur $(\mathrm{H})$ et la période $(\mathrm{T}): \mathrm{H}=3 \mathrm{~m}$ et $\mathrm{T}=7,7 \mathrm{~s} ; \mathrm{H}=7 \mathrm{~m}$ et $\mathrm{T}=11 \mathrm{~s}$. 


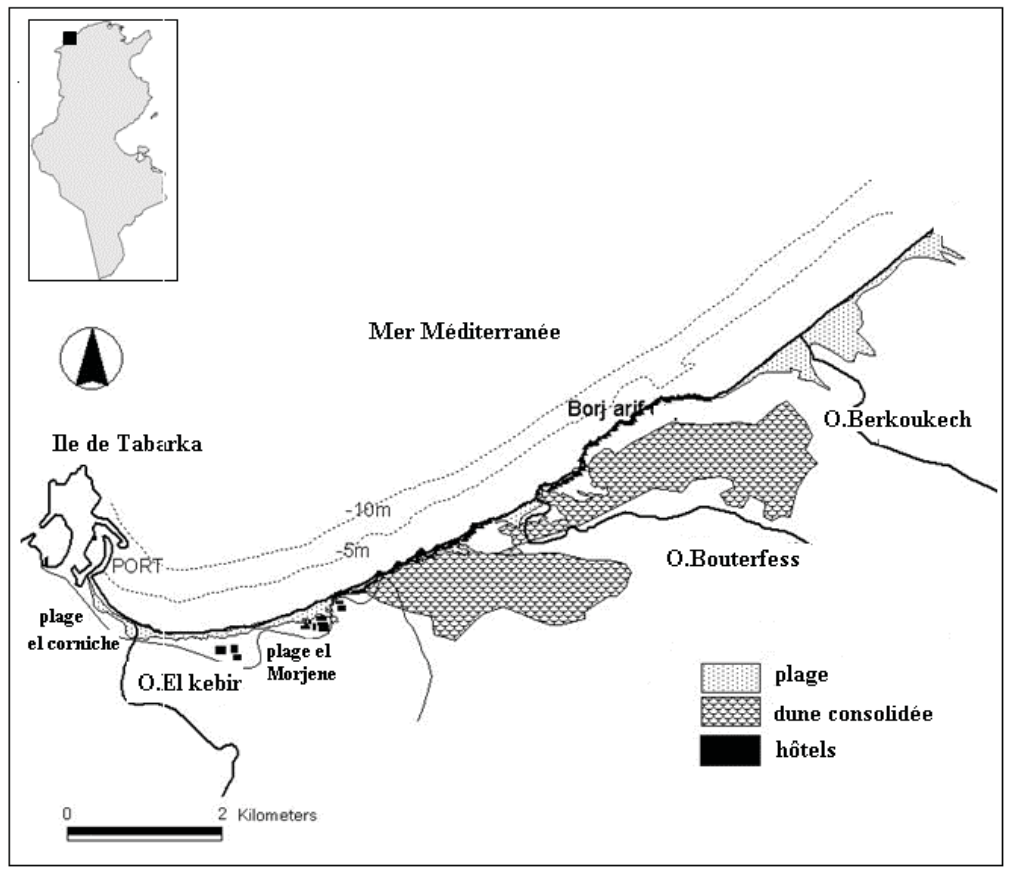

Figure1. Localisation du secteur d'étude.

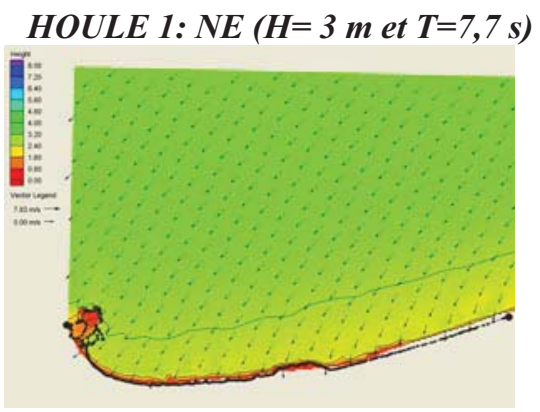

HOULE 2 : NE $(H=7$ m et $T=11$ s $)$
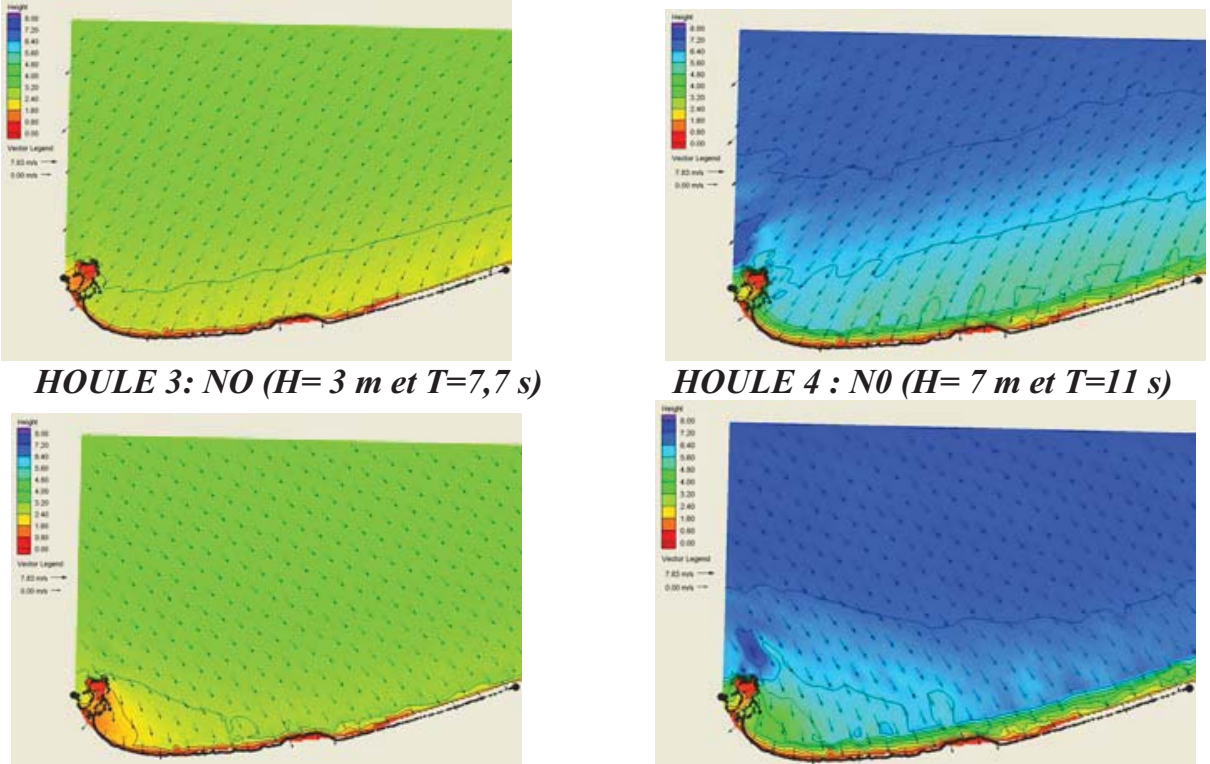

HOULE $4:$ NO $(H=7$ et $T=11 \mathrm{~s})$

Figure 2.Cartes de répartition spatiales de la hauteur de la houle pour les directions NO et NE.

\section{Résultats}

Les résultats de la simulation de la propagation des houles des secteurs Nord-Ouest et Nord-Est, par le modèle STWAVE, pour les deux conditions de la houle (résultats non présentés), montrent que les vecteurs de houle à la côte ont subit une réorientation par rapport à leur direction initiale. Ceci est du au phénomène de réfraction qui affecte la 
houle à l'approche de la côte. En effet, les isobathes deviennent, prés du rivage, plus ou moins parallèles aux lignes de crêtes des houles, avec un rapprochement des lignes orthogonales (par rapport aux lignes de crêtes) au niveau du cap Borj Arif et un éloignement dans le reste du secteur.

En plus du phénomène de réfraction, les houles de secteur Nord ouest subissent une diffraction au niveau de la digue du port de Tabarka, tout en contournant l'île de Tabarka. Ce phénomène se manifeste par un groupe de vecteurs de houle espacées et réorientés vers la terre. Il devient plus important lorsque la hauteur de la houle augmente de 3 à $7 \mathrm{~m}$, ce qui implique la réduction de l'extension spatiale de la zone protégée par la digue. En effet, les houles deviennent plus énergétiques quand la hauteur augmente.

Les houles de direction Nord-Est subissent une faible réfraction comparée aux houles du secteur nord-ouest. Ceci est en rapport avec l'orientation de la ligne de rivage et l'angle d'incidence des houles.

\section{Conclusion}

L'application du modèle STWAVE pour simuler la propagation de la houle du large. vers la côte dans la frange littorale Tabarka-Berkoukech, nous a permis de tirer les principales conclusions suivantes :

- Les modifications des caractéristiques des houles des secteurs NE et NO sont dues principalement au phénomène de réfraction et localement au phénomène de diffraction par la digue du port et "l'île de Tabarka".

- Les vecteurs des houles (orthogonales par rapport aux lignes des crêtes) convergent au niveau du cap Borj Arif et dans la plage El Morjene, ce qui explique une concentration d'énergie et donc une érosion dans ce deux zones. Ceci est confirmé par l'étude diachronique de l'évolution du rivage qui montre que le trait de côte de la plage El Morjene a subi un recul de 5 à 69 m, pendant 26 ans (HALOUANI et al., 2007).

\section{Références bibliographiques}

HP -Hidrothecnica Portugesa- (1995). Etude générale pour la protection du littoral tunisien. Rapport I-II-III-IV et V, Ministère de l'Equipement Tunisien, 300 p.

HALOUANI N., SABATIER F., GUEDDARI M., FLEURY J. (2007). Evolution $d u$ trait de côte de Tabarka-Bouterfess, Nord-Ouest de la Tunisie. Méditerranée $\mathrm{N}^{\circ} 108$, pp 131-137, 2007.

OUESLATI A. (1994). Les côtes de la Tunisie, recherches sur leur évolution au Quaternaire. Doctorat Es-Sciences, F.L.S.H.T., 402 p.

SMITH J.M., SHERLOCK A.R., RESIO D.T. (2001). STWAVE: steady-state spectral WAVE model: user's manual for STWAVE Version 3.0, Supplemental Report ERDC/CHL-SR-01-1. US Army Engineer Research and Development Center, Vicksburg, MS. 